\title{
长江口及其南部邻近地区大弹涂鱼种群 遗传结构及种群历史分析
}

\author{
刘至治，杨金权，王正琦 ${ }^{* *}$, 唐文乔* \\ (上海海洋大学 鱼类研究室, 水产种质资源发掘与利用教育部重点实验室, 上海 200090)
}

\begin{abstract}
摘要: 应用随机扩增多态性DNA（RAPD）技术及细胞色素 $b$ (Cyt $b$ ) 基因序列, 分析了长江口及其南部邻 近地区大弹涂鱼（Boleophthalmus pecinirostris）群体遗传结构及种群历史, 结果显示这一区域大弹涂鱼群体的遗 传多样性水平很高。(1) RAPD分析结果：从 100 个 10 碱基随机引物中篎选出 30 个多态性引物，对长江口九段 沙湿地、浙江舟山定海、福建霞浦群体各 20 个体进行RAPD分析, 3 个群体分别获得 236、270、274 条带, 共 301 个有效位点。3 群体多态位点比例 $(P)$ 、 Nei基因多样性指数 $(H)$ 、Shannon多样性指数 $(I)$ 分别在 $93.02 \%-96.35 \%$ 、 0.3890 - $0.4219 、 0.5618-0.6044$ 。分子方差分析 (AMOVA）表明，大弹涂鱼群体间存在显著的遗传分化 $\left(F_{s t}=\right.$ 0.03004-0.03254, $P<0.05$ ), 但仅 3.2\%的遗传变异来自群体间。（2）Cyt $b$ 基因序列分析结果：从采自九段沙、定

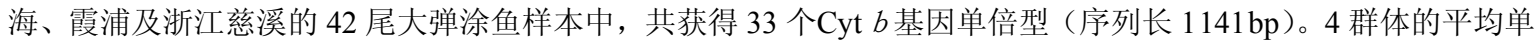
倍型多样性 $(h)$ 、核甘酸多样性 $(\pi)$ 分别为 $0.9814 、 0.0048$ 。 4 群体间的遗传分化指数 $F_{s t}$ 为 $0.00043-0.07814$, 仅 $1.93 \%$ 的变异来自群体间（AMOVA分析），而基因交流值却达 14.50-30.79，群体间K2-P遗传距离为 $0.0040-$ 0.0056 , 从而显示大弹涂鱼群体间没有发生明显的地理分化。以Cyt $b$ 基因序列构建的 NJ树揭示 4 个群体的个体组 成 2 个谱系，但这 2 个谱系与地理分布并不相关。中性检验、错配分析和网络亲缘关系分析皆表明大弹涂鱼群体 有过种群扩张, 扩张时间约在更新世末期的 $0.057-0.023$ 百万年前。
\end{abstract}

关键词: 大弹涂鱼; 细胞色素 $b$; RAPD, 种群遗传结构; 长江口

中图分类号: Q959.483; Q349 文章标识码: A 文章编号: 0254-5853 (2009) 01-0001-010

\section{Genetic Structure and Population History of Beleophthalmus petinirostris in Yangtze River Estuary and Its Southern Adjacent Regions}

\author{
LIU Zhi-zhi, YANG Jin-quan, WANG Zheng-qi**, TANG Wen-qiao* \\ (Laboratory of Fishes, Shanghai Ocean University; Key Laboratory of Exploration and Utilization of Aquatic Genetic \\ Resources, Ministry of Education, Shanghai 200090, China)
}

\begin{abstract}
In order to analysis the genetic structure and demographic history of Boleophthalmus pectinirostris, the random amplified polymorphic DNA (RAPD) technology and mitochondrial cytochrome $b$ (Cyt $b$ ) gene sequences were used in the study. The results showed that there was high level genetic diversity in the populations of $B$. pecinirostris in Yangtze River estuary and its southern wet-land adjacent regions. (1) RAPD was conducted to analysis the genetic variation of B. pecinirostris in Jiuduansha (JDS), Dinghai (DH) and Xiapu (XP) for 20 individuals per population, with 30 polymorphic 10-based random primers selected from 100 ones. In total, 236, 270 and 274 bands were obtained for JDS, DH and XP, respectively and there was 301useful loci. The range of proportion of polymorphic loci $(P)$, Nei's gene diversity $(H)$ and Shannon diversity index $(I)$ was: $93.02 \%-96.3 \%, 0.3890-0.4219,0.5618-0.6044$, respectively. The results of AMOVA showed significantly genetic differentiation $\left(F_{s t}=0.03004-0.03254, P<0.05\right)$ but there was only $3.2 \%$ genetic variation among populations. (2) Results of Cyt $b$ gene: the total Cyt $b$ gene length was $1141 \mathrm{bp}$ and 33
\end{abstract}




\begin{abstract}
haplotypes were obtained from 42 individuals collecting in 4 different regions, which included JDS, DH, XP and Cixi (CX). The average haplotype diversity $(h)$ and nucleotide diversity $(\pi)$ was $0.9184,0.0048$, respectively. The high level of $N_{m}$ values (14.50-30.79) and low $F_{s t}$ values (0.0043-0.07814), the average K2-P distances (0.0040-0.0056), as well as the AMOVA analysis (total $F_{s t}=0.0193, P>0.05$ ) all revealed that the $B$. pecinirostris had no obviously geographical differentiation. However, the phylogenetic tree estimated by neighbor-joining $(\mathrm{NJ})$ method indicated that the individuals of $B$. pecinirostris formed two monophyletic lineages, and the two lineages were not correlated with geographical populations. Neutrality tests, mismatch distribution and network analysis suggested a late Pleistocene population expansion for $B$. pecinirostris (0.057-0.023 million years ago).
\end{abstract}

Key words: Beleophthalmus petinirostris; Cytochrome $b$; RAPD; Population genetic structure; Yangtze River estuary

大弹涂鱼 (Boleophthalmus pectinirostris), 俗称 花跳、星跳、跳鱼等, 隶属于鲈形目 (Perciformes) 鰕虎鱼科(Gobiidae), 为暖水广温广盐性海洋鱼类, 生活在河口及潮间带的淤泥滩涂及红树林区, 在我 国主要分布于江苏、上海、浙江、福建、广东、广 西和台湾沿海。大弹涂鱼不仅是深受环保专家关注 的滩涂湿地指示生物, 而且是我国南方最具利用价 值的滩涂鱼类之一。日本和我国台湾自上世纪 60 年代就开始养殖, 我国东南沿海的养殖则兴起于 20 世纪 90 年代, 已成为当地名特优养殖品种。

近年来, 由于大规模的海岸线开发、围海造地 以及海岸污染, 大幅度地减少并割裂了大弹涂鱼类 的栖息地, 过度的捕捞也造成了其野生种群数量的 严重下降。研发规模化育苗技术并有效养护野生种 群, 已成为大弹涂鱼产业化开发的重要环节, 而探 明其种群遗传结构是实施这一环节的前提。目前, 有关大弹涂鱼的形态特征、行为习性、养殖繁育技 术、性腺发育等已有许多报道 (Zhang et al, 2006), 但在种群组成和遗传结构等方面的研究却很少(Jin et al, 2004; Kanemori et al, 2006; Zhang et al, 2006), 因而开展此方面的研究很有必要。

随机扩增多态性 DNA (RAPD) 是动物遗传 多样性研究的常用技术, 具有安全、快捷、简便、 高效等特点(Liu et al, 2004; Matoso et al, 2004)。另 外一类分子标记, 线粒体 DNA (mtDNA) 以严格 的母系遗传和较快的进化速率, 已成为一种探讨物 种系统发生、种内遗传分化的有效遗传标记 (Verheyen et al, 2003; Wang et al, 2006; Zhu et al, 2007), 而细胞色素 $b$ (Cyt $b$ ) 是 mtDNA 中应用较 多的分析鱼类种群遗传结构的分子片段(Han et al, 2007; Santos et al, 2006; Wang et al, 2006)。长江口及 其邻近的沿岸地区是我国人类活动干预最强烈的 地区, 建立有国家级崇明东滩和九段沙湿地自然保 护区, 大弹涂鱼是这些保护区最具标志意义的滩涂
鱼类之一。本研究旨在通过 RAPD 技术分析与 Cyt $b$ 基因全序列的测定，了解这些地区大弹涂鱼天然群 体的遗传结构与发生历史, 为其资源保育与合理利 用提供基础资料。

\section{1 材料与方法}

\section{1 样本采集与选用}

通过当地渔民设置网具抓捕，于 2005 年 5 月 一 2006 年 8 月采集到一批不同地方的大弹涂鱼天然 群体样本。其中，任取九段沙湿地自然保护区（简 称 JDS)、浙江舟山定海 (简称 DH)、福建霞浦县 官井洋 (简称 XP) 样本各 20 尾, 用于 RAPD 分析; 任取 JDS 样本 11 尾、XP 样本 10 尾、DH 样本 10 尾、浙江慈溪样本 11 尾（简称 $\mathrm{CX}$ ), 用于 $\mathrm{Cyt} b$ 基 因序列测定。另选取采自舟山定海的弹涂鱼

( Periophthalmus modestus)、大鰙弹涂鱼 ( $P$. magnuspinnatus) (Wang et al, 2006)及福建霞浦的青 弹涂鱼（Scartelaos viridis）各 1 尾, 测定其 Cyt $b$ 基因序列作为构建系统发育树的外类群。所有样本 用 $95 \%$ 乙醇固定，一周后保存于 $70 \%$ 乙醇，置 $4^{\circ} \mathrm{C}$ 冰箱备用。取过样的标本保存于上海海洋大学鱼类 标本室。

\section{2 基因组 DNA 提取}

基因组DNA提取采用常规的“酚一氯仿”法, 参 照Liu et al (2004)的方法并做适当修改。具体操作如 下: 取 $0.1 \mathrm{~g}$ 左右背鯺或尾鯺, 切碎, 加入 $495 \mu \mathrm{L}$ 裂 解缓冲液 (Sambrook et al, 2002)， $5 \mu \mathrm{L}$ 蛋白酶K (浓 度为 $20 \mathrm{mg} / \mathrm{mL}$ ), $55-56^{\circ} \mathrm{C}$ 恒温水浴锅消化 $2-3 \mathrm{~h}$ (也可放置过夜, 约 $12 \mathrm{~h}$ )。加入等体积饱和酚, 缓 慢转动 $20 \mathrm{~min}, 12000 \mathrm{r}$ 离心 $10 \mathrm{~min}$ 。吸取上清液, 再加入等体积的氯仿一异戊醇混合液 $(24: 1)$, 缓 慢转动 $5 \mathrm{~min}$ 。取上清液, 加入 2 倍体积的预冷无水 乙醇沉淀DNA $\left(-20^{\circ} \mathrm{C}\right)$, 用 $70 \%$ 乙醇洗涤 1 次, 干 燥, 加入 $300-500 \mu \mathrm{L}$ 的 $\mathrm{ddH}_{2} \mathrm{O}$ 及 $1-2 \mu \mathrm{L} \mathrm{RNA}$ 酶, 
$4{ }^{\circ} \mathrm{C}$ 冰箱保存备用。待 DNA溶解后, 取 $5 \mu \mathrm{L}$ 溶液, $1 \%$ 琼脂糖凝胶电泳检查DNA提取情况。

\subsection{RAPD-PCR 扩增}

10 碱基随机引物 100 个（引物编号为 S1-S60, S101-S140), 购自上海生工生物工程技术服务有限 公司。参照说明书配置浓度 $5 \mu \mathrm{mol} / \mathrm{L},-20^{\circ} \mathrm{C}$ 冰箱保 存备用。扩增条件参照 Liu et al (2004)的方法。取 $10 \mathrm{~mL}$ 扩增产物经 $1 \%$ 进口琼脂糖凝胶 (含 $\mathrm{EB} 0.5$ $\mathrm{mg} / \mathrm{mL}$ ) 电泳 $2 \mathrm{~h}$ 后, 用 Gene-Genius 凝胶成像系统 拍照、记录和分析。

\subsection{Cyt b 基因的 PCR 扩增、纯化及测序}

扩增 Cyt $b$ 基因使用的特异性引物为： L14724

(5' -GAC TTG AAA AAC CAC CGT TG-3' ) 和 H15915 (5' -CTC CGA TCT CCG GAT TAC AAG AC-3' ) (Xiao et al, 2001), 由上海生工生物技术服 务有限公司合成。 PCR 反应在 Eppendorf Mastercycler Gradient PCR 仪上进行, 反应体系为 $25 \mu \mathrm{L}: 10 \times$ buffer $2.5 \mu \mathrm{L}$, dNTP $1 \mu \mathrm{L}(2.5 \mathrm{mmol} / \mathrm{L})$, 引物 L14724、H15915 各 $1 \mu \mathrm{L}(10 \mu \mathrm{mol} / \mathrm{L})$, 模板 DNA $2 \mu \mathrm{L}$ (约 50-200ng), $0.5 \mu \mathrm{L}$ Taq DNA 聚合酶 $(2 \mathrm{U} / \mu \mathrm{L}$ ，购自天根生物科技有限公司 ), $17 \mu \mathrm{L}$ 纯 净水 (购自华舜生物工程有限公司), 加入 $10 \mu \mathrm{L}$ 石 腊油。循环程序为: $95^{\circ} \mathrm{C}$ 预变性 $4 \mathrm{~min} ; 94^{\circ} \mathrm{C} 35 \mathrm{~s}$, $52^{\circ} \mathrm{C} 40 \mathrm{~s}, 72^{\circ} \mathrm{C} 70 \mathrm{~s}, 35$ 个循环; $72^{\circ} \mathrm{C}$ 延伸 $10 \mathrm{~min}$ 。 取 $4 \mu \mathrm{L}$ 扩增产物经 $0.8 \%$ 琼脂糖凝胶 (含 $\mathrm{EB}$ $0.5 \mu \mathrm{g} / \mathrm{mL}$ ) 电泳, 电泳缓冲液为 $5 \times \mathrm{TBE}$ (用时稀释 10 倍), 电压 $5 \mathrm{~V} / \mathrm{cm}$, 电泳 $1 \mathrm{~h}$ 后, 用 Gene-Genius 凝胶成像系统拍照检测。目的基因用 UNIQ-10 柱式 DNA 胶回收试剂盒（上海生工技术服务有限公司） 纯化回收, 送上海生工技术服务有限公司, 由 $\mathrm{ABI}$ 377 自动测序仪进行正反向测序。测序所用引物同 PCR 扩增反应。

\section{5 数据分析}

1.5.1 RAPD 分析 经电泳获得 RAPD 图谱, 在同 一电泳迁移位置上, 有 RAPD 扩增条带的计为 1 , 没有的计为 0 , 每群体所有个体在全部位点上的谱 带式样 (RAPD 表型) 组成一个 (0 或 1 ) 矩阵。用 POPGENE version 1.3.1 软件(Yeh et al, 1999)进行分 析, 计算多态位点数、多态位点比例 $(P) 、 \mathrm{Nei}$ 基 因多样性指数 $(H)$ 、Shannon 多样性指数 $(I)$ 及群 体间遗传距离等。用 Arlequin Ver. 3.01 软件进行分 子方差分析 ( analysis of molecular variance, AMOVA) (Excoffier et al, 2005)。
1.5.2 Cyt $b$ 基因 序列的对位排列（alignment）使 用Clustal X 软件(Thompson et al, 1997), 并在 SEAVIEW程序(Galtier et al, 1996)中辅以手工校正。 采用Mega 4.0 软件(Kumar et al, 2004)分析核苷酸组 成及统计单倍型、计算转换/颠换值 ( $\mathrm{Ti} / \mathrm{TV}$ ) 及 Kimura 双参数遗传距离 (Kimura 2-parameter distance, K2-P), 并用该软件把核苷酸序列转化为 氨基酸序列; 同时采用Mega 4.0 软件的邻接法 (neighbor-joining, NJ) 构建大弹涂鱼各单倍型间系 统发育关系树, 以青弹涂鱼、大鯺弹涂鱼、弹涂鱼 为外类群, 以Kimura双参数法为替代模型, 系统树 分支的置信度采用自展法 (bootstrap analysis, BP) 重复检测 1000 次。用Arlequin Ver. 3.01 软件统计种 群核苷酸多样度 (nucleotide diversity, $\pi$ )、单倍型 多样度 (haplotype diversity, $h$ ) 及其标准差 $(S D)$ 。 采用AMOVA分析方法, 以 1000 次重复随机抽样单 倍型重排后进行显著性检验, 用于估计大弹涂鱼种 群遗传结构及不同地理种群遗传变异的分布。用 DnaSP Ver. 4.10 软件(Rozas et al, 2006)对群体间分 化指数 $\left(F_{s t}\right)$ 、基因交流值 $\left(N_{m}\right)$ 及 Cyt $b$ 基因单倍 型错配进行分析。为了解大弹涂鱼的种群历史, 以 Arlequin Ver. 3.01 软件进行Tajima's D中性检验。以 TCS 软件(Clement et al, 2000)建立网状亲缘关系 (network)。

根据公式 $T=D_{\mathrm{xy}} / 2 \mu$ 计算谱系间分化时间, $\mu$ 是核 苷酸的变异率速率（1\%-2.5\%) /百万年（Irwin et al ，1991; Martin et al ，1992)。用公式 $\tau=2 u t$ 估算 种群扩张时间 T(Roges et al, 1992)， $T=t \times$ 代时, 代 时由研究对象的性成熟年龄决定。其中, $t$ 表示种群 扩张至今所经历的代数, $\tau$ 是错配分布的模型, 可由 Arlequin3.01 软件分析获得, $u$ 是所测Cyt $b$ 序列总的 变异率, 用公式 $u=2 \mu K$ 计算 $(K$ 是序列中核苷酸的数 量)。

\section{2 结 果}

\subsection{RAPD 分析}

本研究从 100 个 10 碱基随机引物中, 篮选出 多态性丰富的引物 30 个: S5、S11、S12、S13、S27、 S28、S37、S43、S45、S47、S102、S105、S107、 S108、S112、S114、S117、S119、S122、S123、S124、 S125、S126、S127、S128、S129、S132、S137、 S139、S140。对于 JDS、DH、XP 3 群体, 每群体 各扩增 20 个体。每个引物都能扩增出 6-13 条清 
晰而重复性好的条带 (图 1)。30 个引物在 JDS、 DH、XP 群体分别扩增出 236、270、274 条带, 共 301 个有效位点。每个引物在不同群体内平均扩增 出 7.86-9.13 条带, 其中多态性条带达 90\%以上。
DNA 扩增大小在 $0.3-3.0 \mathrm{~kb}$ 。

3 个群体大弹涂鱼的多态位点比例 $(P) 、 \mathrm{Nei}$ 基因多样性指数 $(H)$ 和 Shannon 多样性指数 $(I)$ 分别在 $93.02 \%-96.35 \% 、 0.3890-0.4219$ 和 0.5618

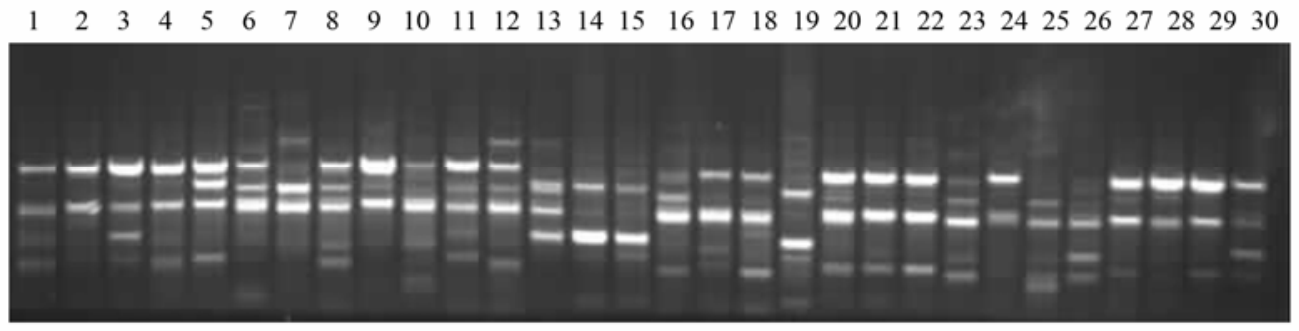

图 1 引物 S126 在大弹涂鱼 3 群体中扩增结果

Fig. 1 Amplified result of primer S126 in JDS, DH and XP populations of Boleophthalmus pectinirostris JDS: 1-10; DH: 11-20; XP: 21-30.

表 1 大弹涂鱼群体间的多态位点比例（P)、Nei 基因多样性 $(\boldsymbol{H})$ 和 Shannon 多样性指数 $(I)$

Tab. 1 Polymorphic loci $(P)$, Nei's gene diversity $(H)$ and Shannon diversity index $(I)$ among populations of Boleophthalmus pectinirostris

\begin{tabular}{lccc}
\hline & 九段沙 JDS & 定海 DH & 霞浦 XP \\
\hline 多态位点数 Polymorphic loci & 281 & 290 & 280 \\
多态位点比例 $(P, \%)$ Propotion of polymorphic loci $(P, \%)$ & 93.36 & 96.35 \\
Nei 基因多样性 (H, 平均值士标准误) Nei's gene diversity (H, mean $\pm S E)$ & $0.3890 \pm 0.1421$ & $0.4219 \pm 0.1086$ & $0.3891 \pm 0.1430$ \\
Shannon 多样性指数 (I, 平均值士标准误) Shannon diversity index(I, mean $\pm S E)$ & $0.5618 \pm 0.1890$ & $0.6044 \pm 0.1418$ & $0.5619 \pm 0.1897$ \\
\hline
\end{tabular}

-0.6044。由表 1 知, 大弹涂鱼群体的遗传多样性 都很丰富, 其中DH群体最高, 而JDS、XP群体之间 比较接近, 却低于DH群体。对 3 个群体间的遗传 差异进行AMOVA分析表明, 总的遗传分化指数 $F_{s t}$ 值为 0.03196 , 群体间 $F_{s t}$ 值在 $0.03004-0.03254$ $(P<0.05)$, 存在显著的遗传分化。在遗传差异的总 变异中, 有 $96.80 \%$ 的差异来自各群体内部, 仅 $3.20 \%$ 的差异来自群体间（表 2)。3 个群体内的遗传相似 度都很高, 在 $0.9403-0.9553$, 而彼此间的遗传距 离却不大, JDS 与 DH、XP间的遗传距离分别为 $0.0457 、 0.0543, \mathrm{XP}$ 与 DH间的遗传距离为 0.0616 。

\subsection{Cyt $b$ 基因序列分析}

2.2.1 序列变异 本实验中, 获得的 Cyt $b$ 基因的 核甘酸序列长度为 $1141 \mathrm{bp}$, 最后一个碱基为 $\mathrm{T}$, 在 线粒体上为不完全终止密码子 $U$ (Anderson et al, 1981)。经 MEGA 4.0 软件分析, 从 42 尾大弹涂鱼 中共获得 33 个 Cyt $b$ 基因单倍型, GenBank 序列号 为 EU503190-EU503222。每群体分别有 8-10个 单倍型, 其中 $\mathrm{H} 2 、 \mathrm{H} 4 、 \mathrm{H} 5$ 是共享单倍型, 以 $\mathrm{H} 2$ 共享频率和出现次数最多 (5 尾个体, 占测序样本 总数的 $11.9 \%$ ), 其余的 30 个为各群体特有的单倍
型（表 3)。碱基组成中, $\mathrm{A} 、 \mathrm{~T} 、 \mathrm{C} 、 \mathrm{G}$ 含量分别为 $26.1 \% 、 32.1 \% 、 27.7 \% 、 14.1 \%, \mathrm{~A}+\mathrm{T}$ 含量为 $58.2 \%$, $\mathrm{G}+\mathrm{C}$ 含量为 $41.8 \%$, 表现出明显的反 $\mathrm{G}$ 偏倚。在 1141 个序列位点中未发现插入和缺失, 其中保守位 点有 1083 个、变异位点 58 个 (图 2)。序列的平均 转换与颠换 $(T i / T v)$ 比值为 4.0 , 密码子第三位点 的 $T i / T v$ 值为 7.9, 显示变异主要发生在该位点, 而 密码子第一、第二位点的 $\mathrm{Ti} / \mathrm{TV}$ 值较小, 分别为 2.2 和 0.4 。4 个群体内 Kimura 双参数 (K2-P) 遗传距 离为 $0.0030-0.0058$, 群体间为 $0.0040-0.0056$, 二者比较接近, 只有定海与其他 3 个群体间的遗传 距离都高于群体内（表 4)。

在氨基酸水平上, 若以 $\mathrm{H} 2$ 的序列为参照, 则 所有单倍型中发生变异的位点仅 15 个, 即第 3 位 Ser 变为 Ile 或 Cys, 第 20 位 Asp 变为 Glu、Asn 或 Cys, 第 21 位 Leu 变为 His, 第 22 位 Pro 变为 Gln, 第 130 位 Gly 变为 Cys, 第 135 位 $\mathrm{Val}$ 变为 $\mathrm{Ala}$, 第 166 位 Gly 变为 Ser, 第 221 位 His 变为 Pro, 第 237 位 The 变为 Asn, 第 304 位 Val 变为 Ile, 第 307 位 Leu 变为 Pro, 第 311 位 Gln 变为 His, 第 312 位 $\mathrm{Arg}$ 变为 Pro, 第 376 位 Leu 变为 $\mathrm{Val}$, 第 377 位 Leu 
变为 His (未列出氨基酸序列比较图)。

2.2.2 种群遗传结构 4 个大弹涂鱼群体的单倍型 多样性 $(h)$ 、核苷酸多样性 $(\pi)$ 值分别在 0.9556
$-0.9818 、 0.0030-0.0058$, 平均值为 $0.9814 、 0.0048$, 其中 XP 群体呈现出最低的单倍型多样性却显现出 最高的核甘酸多样性（表 3)。利用 DnaSP 估算大

表 2 大弹涂鱼群体间遗传差异的分子方差分析（AMOVA）

Tab. 2 Analysis of molecular variance(AMOVA) in populations of Boleophthalmus pectinirostris

\begin{tabular}{lccccccc}
\hline \multirow{2}{*}{ 变异来源 Source of variation } & \multicolumn{2}{c}{ 自由度 $d f$} & \multicolumn{2}{c}{$\begin{array}{c}\text { 变异组成 } \\
\text { Variance components }\end{array}$} & \multicolumn{2}{c}{$\begin{array}{c}\text { 变异百分比 } \\
\text { Percentage of variance }\end{array}$} \\
\cline { 2 - 8 } & Cyt $b$ & RAPD & Cyt $b$ & RAPD & Cyt $b$ & RAPD \\
\hline 群体间 Among pop & 3 & 2 & 0.053 & 1.84 & 1.93 & 3.20 \\
群体内 Within pop & 29 & 57 & 2.706 & 55.85 & 98.07 & 96.80 \\
\hline 总变异 Total variation & 32 & 59 & 2.759 & 57.69 & 100.00 \\
\hline
\end{tabular}

11111111

1122333334444445556666666666777788899999999900001111

56664611125892366998990112466679111812501223356624891123

7880254869875836458682246256226983057338830016846997911360 A GGC T C C C TAAT C G CA T T GGA T CAAACG TAAC C C C C GA GC C T G T TAGAACA C C G T G C T

$\ldots \ldots$. . . . . . . . . . . . . . . . . . . . . . . . .

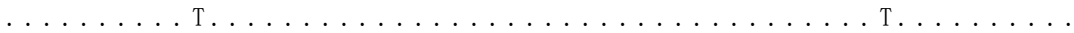

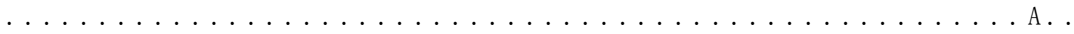

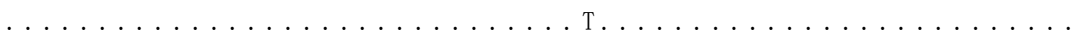

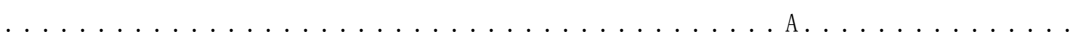

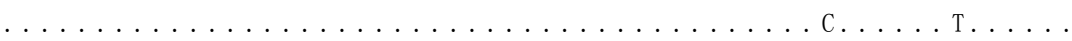

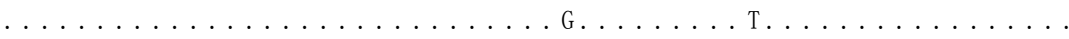

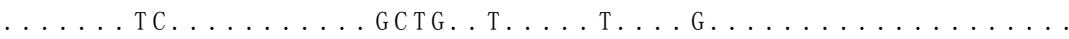

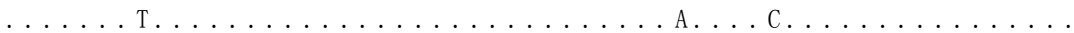

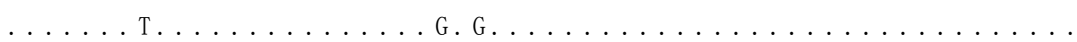

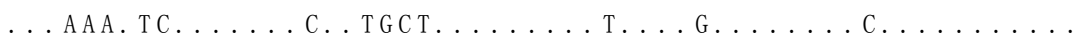

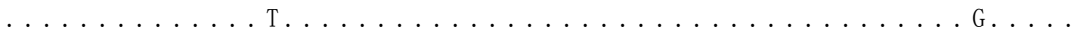

$\ldots \ldots$. . . . . . . G. . . . . . . . . . . .

$\ldots \ldots$. . . ...........................

$\ldots \ldots$. . . . с . . . . . . . . . . . . .

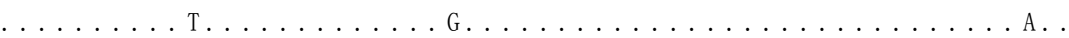

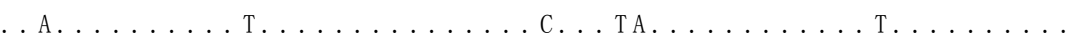

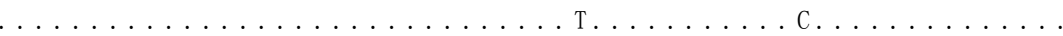

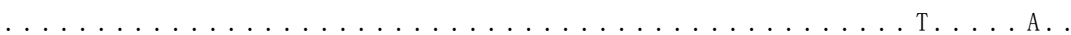

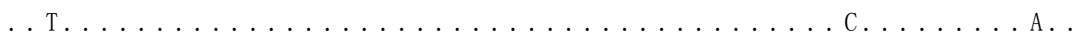

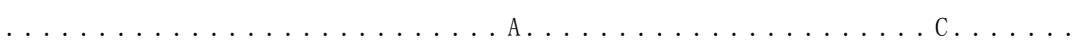

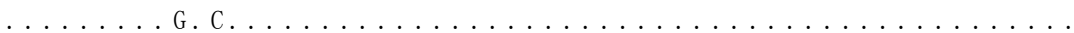

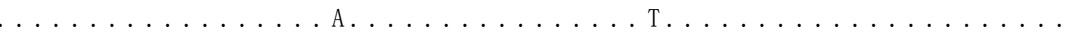

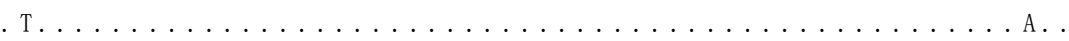

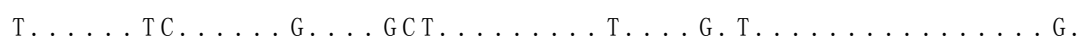

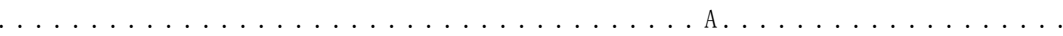

$\ldots \ldots \ldots \ldots \ldots$ G. . . . . . . . . . . . . . . . . . . . . .

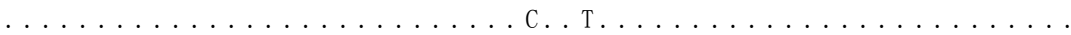

$\ldots \ldots$. . . . . . . . . . . . . . . . .

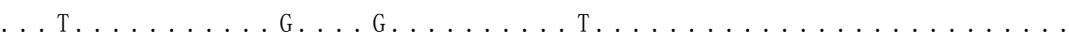

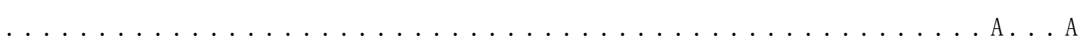

$33 \ldots \ldots$ т....... . . . . . . . . . . . . .

图 24 个大弹涂鱼群体的 33 个 Cyt $b$ 基因序列单倍型的变异位点比较

Fig. 2 Variable sites of Cyt $b$ sequence among four populaitons of Boleophthalmus Pectinirostris 图上数字由上至下为碱基变异位点在所测序列中的位置。

Numbers at the top of the figure indicate the variable sites in the sequece analysis based on $\mathrm{H} 2$. 
弹涂鱼群体间分化指数 $F_{s t}$ 值与群体基因交流值 $\left(N_{m}\right)$, 结果表明, 大弹涂鱼 4 个群体间的 $F_{s t}$ 值仅 为 $0.00043-0.07814$, 总的群体分化指数 $F_{s t}$ 值为 $0.01932 （ P>0.05)$; 群体间的基因交流值 $\left(N_{m}\right)$ 均 远大于 1 (14.50-30.79), 其中, XP与JDS、CX、 DH 3 群体间的基因交流值（14.50-21.65）较小, 而JDS与CX、DH 2 个群体间的基因交流值（27.00 -30.79) 较大（表 5)。AMOVA分析的结果显示, 98.07\% 的变异来自种群内, 而种群间的变异仅占 1.93\%（表 2)。

以邻接法 $(\mathrm{NJ})$ 构建系统发育树 (外群为青弹 涂鱼、弹涂鱼、大鳍弹涂鱼的Cyt $b$ 基因序列, GenBank序列号分别为: EU503223-EU503225) 显示, 大弹涂鱼 4 个群体可以分为两个大谱系 (lineage) 和多个支系。支系A有 28 个单倍型构成, 支持率为 $52 \%$; 支系B由 5 个单倍型构成, 支持率 为 $78 \%$ 。但这两个谱系并非以 4 个群体按地理单元
分别聚类, 而是每个谱系都包括其他群体的个体 (图 3)。两谱系间的序列差异 $\left(D_{\mathrm{xy}}\right)$ 为 0.01 , 经 计算可得 $T$ 值为 $0.5-0.2$ 百万年 (MY)。此期为地 质时代上更新世晚期, 说明大弹涂鱼在这期间产生 了遗传结构上的差异，从而呈现出现今 cyt $b$ 基因序 列差异较大的两个谱系。

2.2.3 种群历史 利用 Arlequin3.01 软件, 对 4 个 群体分别进行 Tajima's D 中性检验, 因各群体样本 数较少 $(10-11)$, Tajima's D 值均为负值 $(-1.542$ $-0.702)$ 且未达到显著水平 $(P>0.10)$, 故将所有 个体作为一个整体进行分析, 发现 Tajima's $D$ 值 $(D=-2.111)$ 达到显著水平 $(P<0.05)$ 。说明大弹涂 鱼曾作为一个整体经历过种群扩张 (population expansion)。

Network 的结果显示 (图 4), 大弹涂鱼所有的 Cyt $b$ 单倍型拥有一个共享的中心单倍型 (H2), 并 聚成两个分支, 其中采自 XP 的 H1、H26 与采自 JDS

表 3 大弹涂鱼群体样本数、单倍型 $(\boldsymbol{H})$ 、单倍型多样性 $(\boldsymbol{h})$ 和核苷酸多样性 $(\boldsymbol{\pi})$ 值

Tab. 3 Number of sample size and haplotypes, haplotype diversity $(h)$ and nucleotide diversity $(\pi)$ of the four Boleophthalmus pectinirostris populations on Cyt b gene

\begin{tabular}{|c|c|c|c|c|}
\hline 采样地 Populations & $\begin{array}{c}\text { 样本数 } \\
\text { Sample } \\
\text { size }\end{array}$ & 单倍型 Haplotypes(H) & $\begin{array}{c}\text { 单倍型多样性 } \\
\text { Haplotype } \\
\text { diversity }(h)\end{array}$ & $\begin{array}{c}\text { 核甘酸多样性 } \\
\text { Nucleotide } \\
\text { diversity }(\pi)\end{array}$ \\
\hline 上海九段沙 JDS & 11 & $\underline{\mathrm{H}} 2$ (2) $*, \underline{\mathrm{H}} 4, \mathrm{H} 6, \mathrm{H} 7, \mathrm{H} 8, \mathrm{H} 9, \mathrm{H} 10, \mathrm{H} 11, \mathrm{H} 12, \mathrm{H} 13$ & $0.9818 \pm 0.0463$ & $0.0053 \pm 0.0031$ \\
\hline 浙江慈溪 CX & 11 & $\mathrm{H} 2, \underline{\mathrm{H} 5}$ (2) *, H14, H15, H16, H17, H18, H19, H20, H21 & $0.9818 \pm 0.0463$ & $0.0048 \pm 0.0028$ \\
\hline 福建霞浦 XP & 10 & $\mathrm{H} 1(2) *, \mathrm{H} 3(2) *, \mathrm{H} 4, \mathrm{H} 22, \mathrm{H} 23, \mathrm{H} 24, \mathrm{H} 25, \mathrm{H} 26$ & $0.9556 \pm 0.0594$ & $0.0058 \pm 0.0034$ \\
\hline 舟山定海 DH & 10 & $\mathrm{H} 2$ (2), H5, H27, H28, H29, H30, H31, H32, H33 & $0.9778 \pm 0.0540$ & $0.0030 \pm 0.0019$ \\
\hline 总计 Total & 42 & 33 & $0.9814 \pm 0.0118$ & $0.0048 \pm 0.0026$ \\
\hline
\end{tabular}

$\mathrm{H}($ ) *内数字表示某群体中, 出现该单倍型的样本数; $\mathrm{H}$ 表示共享单倍型。

Figure in the brackets shows the number of specimen in the population, $\underline{H}$ indicates the shared haplotypes.

表 4 大弹涂鱼群体内与群体间的 K2-P 遗传距离

Tab. 4 The average K2-P distance among and within the four Boleophthalmus pectinirostris populations

\begin{tabular}{lllll}
\hline 群体 Pop & 九段沙 JDS & 慈溪 CX & 霞浦 XP & 定海 DH \\
\hline 九段沙 JDS & 0.0054 & & & \\
慈溪 CX & 0.0051 & 0.0048 & & \\
霞浦 XP & 0.0056 & 0.0053 & 0.0058 & \\
定海 DH & 0.0044 & 0.0040 & 0.0048 & 0.0030 \\
\hline
\end{tabular}

表 5 大弹涂鱼群体间的基因交流值 $N_{m}$ (对角线上方) 与种群分化指数 $F_{s t}$ (对角线下方)

Tab. 5 Matrix of pairwise Nm (above diagonal) and Fst (below diagonal) among the four Boleophthalmus pectinirostris populations

\begin{tabular}{lcccc}
\hline \multirow{2}{*}{$F_{S T}$} & \multicolumn{4}{c}{$N_{m}$} \\
\cline { 2 - 5 } & 九段沙 JDS & 慈溪 CX & 霞浦 XP & 定海 DH \\
\hline 九段沙 JDS & & 27.00 & 21.65 & 30.79 \\
慈溪 CX & 0.00043 & & 15.41 & 30.79 \\
霞浦 XP & 0.0056 & 0.00513 & & 14.50 \\
定海 DH & 0.04181 & 0.01102 & 0.07814 & \\
\hline
\end{tabular}




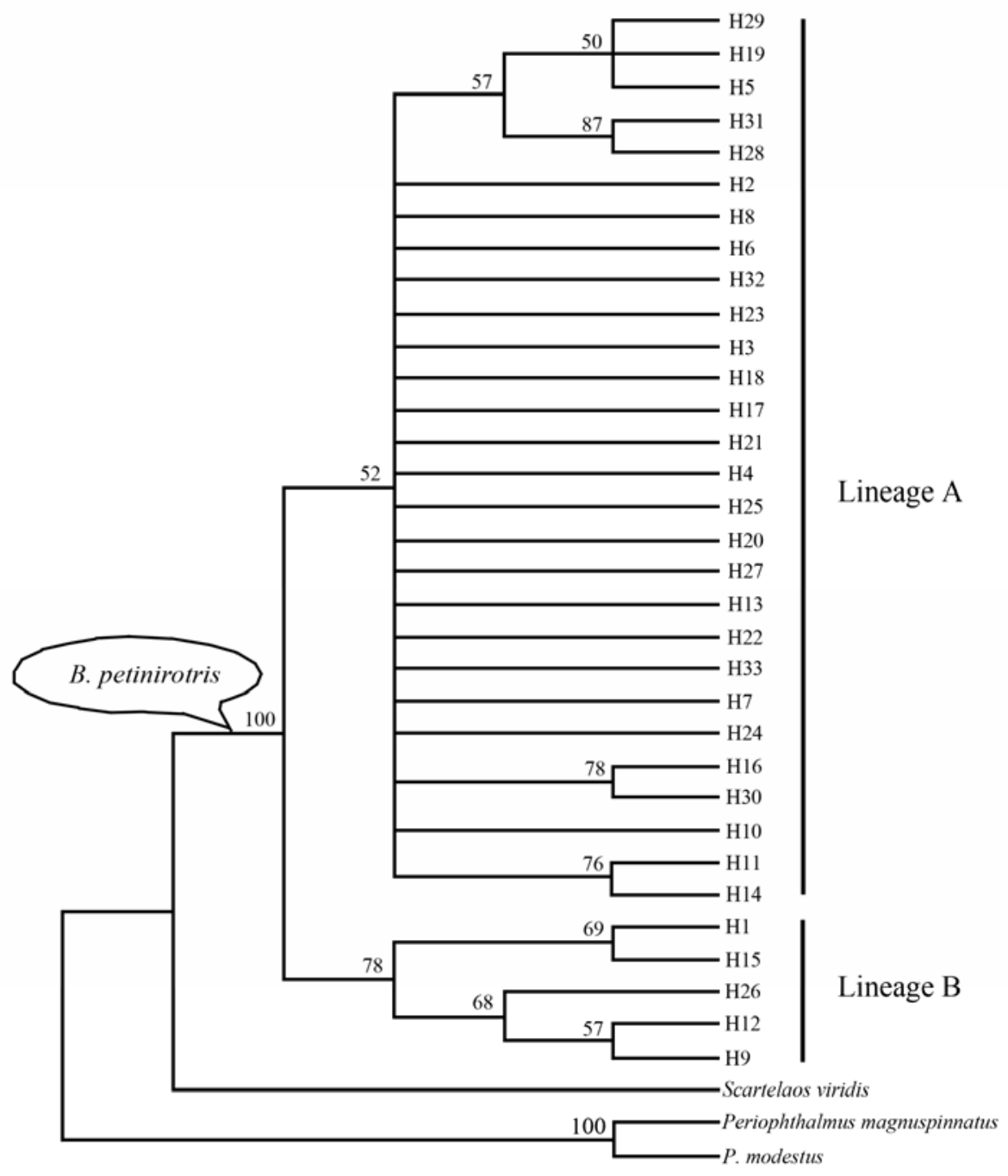

图 3 基于 Cyt $b$ 基因序列构建的 4 个大弹涂鱼群体邻接树

Fig. 3 Neighbor-joining tree based on Cyt $b$ gene sequence variation of four Boleophthalmus pectinirostris population

节点处的数值为 1000 次 bootstrap 检验的支持率，仅显示支持率大于 $50 \%$ 的数据。

Numbers at the nodes indicate bootstrap values with 1000 replicates, only $>50 \%$ shown.

的 H9、H12 及采自 CX 的 H15 单独形成一个分支, 其余 28 个单倍型聚成另一分支, 但 4 个群体的单 倍型并没有依采样地形成各自的分支 (clade)，而 呈现出一个非典型星状（star-like）的发散图。用 DnaSP4.0 对 Cyt $b$ 单倍型进行错配分析, 结果显示, 所有的 Cyt $b$ 单倍型呈现为一条虽不完全平滑但也 只有一个明显顶峰的单峰型曲线 (图 5)。这些同样 说明, 大弹涂鱼历史上有过种群扩张。本研究中, $\tau=2.587, K=1141$, 代时取 1 (因大弹涂鱼通常在 1 龄即可达到性成熟), 计算得到 $T$ 值为 $0.057-$ $0.023 \mathrm{MY}$, 说明大弹涂鱼群体扩张时间约为更新世 晚期。

\section{3 讨 论}

\section{1 大弹涂鱼群体的遗传多样性}

遗传多样性不仅是形成生物多样性的基础，也 是物种进化潜能的保证。遗传多样性的下降, 可能 导致物种对环境适应能力的降低, 这对生活在野外 多变环境中的群体来说是一个极大的威胁 (Vrijienhoek et al, 1994; Frankham et al, 2002)。以往 关于大弹涂鱼遗传多样性的报道很少 (Jin et al, 2004; Kanemori et al; 2006; Zhang et al, 2006), Jin et al(2004)通过 RAPD 的研究结果显示, 浙江宁海三 门湾海区的大弹涂鱼群体的遗传多样性水平比较 
低, 多态位点比例 $(P)$ 仅 $21 \%$, 呼吁加强大弹涂 鱼种质资源的保护。但本研究通过 RAPD 技术及 Cyt $b$ 基因序列分析却显示, 长江口及其南部邻近 的舟山、霞浦等地区的大弹涂鱼天然群体的遗传多 样性却很丰富。RAPD 结果显示, JDS、DH、XP 群体的多态位点比例 $(P)$ 在 $93.02 \%-96.35 \%$ 间, 远远高于三门湾海区大弹涂鱼群体的 $21 \%$, 也比野 生的小黄鱼 (Larimichthys polyactis) 群体的 $91.03 \%$ 及半滑舌鳎 (Cynoglossus semilaevis Güntuer) 的 $76 \%-80 \%$ 高(Han et al, 2007), 显示出很丰富的遗 传多样性。同样, 本研究从 42 尾大弹涂鱼样本中 获得了 Cyt $b$ 基因全序列的单倍型 33 个, 平均单倍 型多样性 $(h)$ 为 0.9184 , 与东海和黄海的日本鯷

(Engraulis japonicus) $(h=0.958)$ 相当 (Yu et al, 2005), 表现出丰富的遗传多样性。可见, 无论从线 粒体 DNA 还是从核基因组 DNA 角度, JDS、DH、

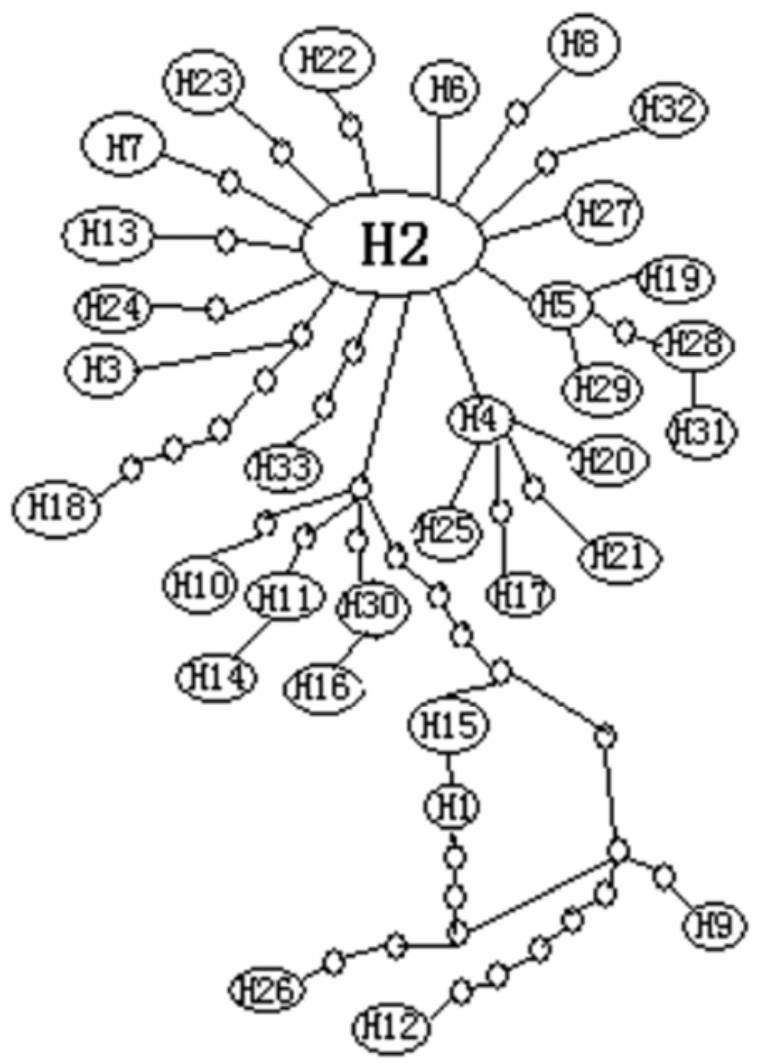

图 44 个大弹涂鱼群体 Cyt $b$ 基因序列单倍型间的网 状亲缘关系图

Fig. 4 Minimum spanning networks showed genetic relationship among haplotypes of Cyt $b$ gene for the four Boleophthalmus pectinirostris populations

H1-H33 表示单倍型, 空心小圆圈表示缺失的中间单倍型。

H1-H33 indicated haplotypes and small empty circles showed the haplotypes that didn't present in the samples but necessary to link all observed ones in the study.

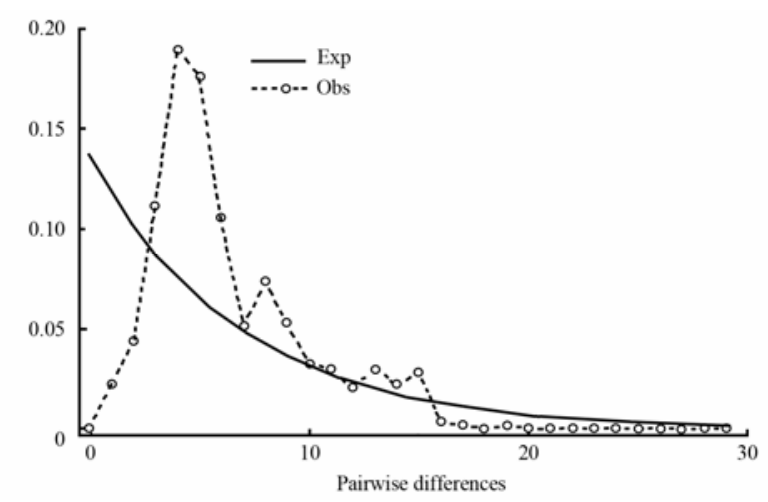

图 54 个大弹涂鱼群体 Cyt $b$ 基因序列单倍型错配分布图

Fig. 5 Observed pairwise sequence difference and the expected mismatch distribution under the model of population expansion on the Cyt $b$ gene haplotypes for the four Boleophthalmus pectinirostris populations

CX、XP 野生大弹涂鱼群体的遗传多样性都很高, 具有较高的进化潜力, 这为开展不同地理种群的种 内杂交和选育提供了种质保障。

\section{2 种群遗传结构及种群历史}

种群分化指数 $\left(F_{s t}\right)$ 常用来表示两个种群间的 遗传分化程度, 在 0 到 1 的范围内, $F_{s t}$ 值越大, 两 种群的分化程度越高(Wright et al, 1978)。种群基因 交流值 $\left(N_{m}\right)$ 用来表示种群间的基因交流程度, 若 $N_{m}>4$, 则表明群体间是一随机交配的群体(Hartl et al, 1989)。本研究Cyt $b$ 基因序列分析显示，除了 DH 与XP群体间的Fst值（0.07814）达到中等分化程度 外, 其他群体间的 $F_{s t}$ 值均很小 $(0.00043-0.04181)$ $(P>0.05)$, 而 $N_{m}$ 值却远大于 $4(14.50-30.79)$ 。从 群体间K2-P遗传距离来看 (表 4), 除DH与其它 3 群体间的距离都高于群体内外, 其余群体间的平均 遗传距离与群体内接近。AMOVA分析表明, 仅 1.93\%的遗传变异来自群体间。这些结果均表明, 本研究所分析的 4 个大弹涂鱼群体间的遗传分化程 度较低, 各采样点群体间基因交流频繁。DH、CX、 JDS之间的基因交流值很大（27.00-30.79），可能 与这三个采样地彼此间的地理位置较近有关。但 RAPD分析结果却显示JDS、DH、XP 3 群体间存在 显著的遗传分化 $(P<0.05)$, 表明在群体遗传差异的 检测上, RAPD比Cyt $b$ 基因更为灵敏, Han et al (2007)也发现同样的现象。Cyt $b$ 基因是线粒体上的 一个基因位点, 它是蛋白质编码基因, 进化速率受 到限制; 而RAPD技术分析的是核基因，其遗传方 式与线粒体基因不同，且RAPD能对整个基因组的 
多个位点的遗传变异信息进行扫描, 这可能是导致 两种分析方法得出不同遗传分化结论的原因。

以邻接法 $(\mathrm{NJ})$ 构建的大弹涂鱼 Cyt b 单倍型 系统发育关系树（图 3) 及网状亲缘关系图（图 4) 均表明, 4 个群体可聚为两个谱系, 但这两个谱系 与地理分布不相关, 表明大弹涂鱼正处于谱系重排 (lineage sorting) 阶段, 这与 Kanemori et al(2006) 的研究结果相似: 他们通过线粒体控制区序列分 析, 发现日本有明海、九州八代湾的大弹涂鱼群体 虽存在一定程度的遗传分化, 但并没有按采样点的 地理单元分开。类似的现象在钩牙黄石首鱼

( Macrodon ancylodon)、弯耙鲱 (Ethmalosa fimbriata) 等海水鱼类也存在(Durand et al, 2005; Santos et al, 2006)。至于引起两个谱系群体大小差 异的原因, 尚需更深入的研究种群历史来解释。

Tajima's $D$ 中性检验常用以推测种群曾经历的 历史事件。如果 Tajima's $D$ 呈负值, 且在统计学上 达到显著标准, 则说明序列中含有比中性进化模型

\section{参考文献:}

Anderson S, Bankier AT, Barrell BG, Bruijn de MHL, Couson AR, Drouin J, Eperon IC, Nierlich DP, Roe BA, Sanger F, Schreier PH, Smith AJH, Staden R, Young IG. 1981. Sequence and organization of the human mitochondrial genome [J]. Nature, 290: 457-465.

Clement M, Posada D, Crandall KA. 2000. TCS: A computer program to estimate gene genealogies [J]. Mol Ecol, 9: 1657-1659.

Durand JD, Tine M, Panfili J, Thaw OT, Laë R. 2005. Impact of glaciations and geographic distance on the genetic structure of a tropical estuarine fish, Ethmalosa fimbriata (Clupeidae, Bwodich S,1825) [J]. Mol Phyl Evol, 36: 277-287.

Excoffier L, Laval G, Schneider S. 2005. Arlequin ver. 3.0: An integrated software package for population genetics data analysis [J]. Evol Bioinf Online, 1: 47-50.

Frankham R, Ballou JD, Briscoe DA. 2002. Introduction to Conservation Genetics [M]. Cambridge: Cambridge University Press.

Galtier N, Gouy M, Gautier C. 1996. SEAVIEW and PHYLO-WIN: Two graphic tools for sequence alignment and molecular phylogeny [J]. Comp Appl Biosci, 12: 543-548.

Hartl DL, Clark AG. 1989. Principle of Population Genetics, 2rd ed [M]. Sunderland: Sinauer Press.

Han ZQ, Zhuang ZM, Gao TX, Liu JX, Li YH, Wang ZY, Tang QS. 2007. Genetic diversity in Cynoglossus semilaevis by AFLP, RAPD and mtDNA markers [J]. J Fish Sci China, 14(2): 192-200. [韩志强, 庄志 猛, 高天翔, 刘进贤, 李玉晖, 王志勇, 唐启升. 2007. 半滑舌鳎 DNA 的群体遗传变异. 中国水产科学, 14 (2) : 192-200.]

Irwin DM, Kocher TD, Wilison AC. 1991. Evolution of the cytochrome b gene of mammals [J]. Mol Evol, 32: 128-144.

Jin CH, Zhong AH, Huang FY, Zhang CD, Li MY. 2004. The research on genetic diversity of Boleophthalmus pectinirostris L. from natural population by RAPD method [J]. Marine $S c i$, 28(12): 26-30. [金春华,
中更多的核苷酸位点变化, 可能预示着该种群 曾经有过扩张的历史。本研究中, 将 4 群体所有个 体混合计算后发现 $D$ 值为负值且显著, 说明大弹涂 鱼在历史上是呈现种群扩张的。Network 分析结果 也同样支持这一观点。我们估计种群扩张时期约在 0.057 - 0.023 百万年前, 处于更新世晚期。许多研 究结果表明, 海洋鱼类的种群扩张也大多处于这一 时期(Liu et al, 2006a; Liu et al, 2006b)。第四纪末期 的几次冰期与间冰期旋回, 曾引起海平面的剧烈升 降, 最剧烈时海平面下降达 120-140 m, 现生海洋 物种的分布及种群遗传结构大多受到这种冰期旋 回的影响(Liu et al, 2006a, b)。因此, 大弹涂鱼可能 经历了同样的种群进化历史。

致谢：上海九段沙湿地自然保护署陈秀芝科长 与郑麟工程师、浙江海洋学院赵盛龙教授及霞浦县 渔民郑银锋等协助采集标本, 上海海洋大学 2003 级本科生戴晨露、陆娇、曹刘晏及唐莉婷四位同学 参加了部分工作，特此一并致谢。

钟爱华, 黄福勇, 张春丹, 李明云. 2004. 大弹涂鱼自然种群遗传多 样性的 RAPD 分析. 海洋科学, 28 (12) : 26-30.]

Kanemori Y, Takegaki T, Natsukari Y. 2006. Genetic population structure of Boleophthalmus pectinirostris inferred from mitochondrial DNA sequences [J]. Jap J Ichthyol, 53: 133-141.

Kumar S, Tamura K, Nei M. 2004. MEGA3: Integrated software for Molecular Evolutionary Genetics Analysis and sequence alignment [J]. Brief Bioinf, 5: 150-163.

Liu JX, Gao TX, Zhuang ZM, Jin XS, Yokogawa K, Zhang YP. 2006a. Late Pleistocene divergence and subsequent population expansion of two closely related fish species, Japanese anchovy (Engraulis japonicus) and Australian anchovy (Engraulis australis) [J]. Mol Phyl Evol, 40: 712-723.

Liu JX, Gao TX, Yokogawa K, Zhang YP. 2006b. Differential population structure and demographic history of two closely related fish species, Japanese sea bass (Lateolabrax japonicus) and spotted sea bass (Lateolabrax maculates) in Northwestern Pacific [J]. Mol Phyl Evol, 39: 799-811.

Liu ZZ, Cai WQ,Li SF. 2004. Analysis of genetic variation of five populations in Trionyx sinensis by RAPD [J]. J Fish China, 28(2): 119-126. [刘至治, 蔡完其, 李思发. 2004. 中华鳖五群体遗传变异 的 RAPD 分析. 水产学报, 28 (2) : 119-126.]

Martin AP, Naylor GJP, Palumbi SR. 1992. Rates of mitochondrial DNA evolution are slow compared with mammals [J]. Nature, 357: 153-155.

Matoso DA, Artoi RF, Galeti PM. 2004. Genetic diversity of the small characid fish Astyanax sp., and its significance for conservation [J]. Hydr, 527(1): 223-225.

Rogers AR, Harpending H. 1992. Population growth makes waves in the distribution of pairwise genetic differences [J]. Mol Boil Evol, 9: 552-569. 
Rozas J, Sánchez-DelBarrio JC, Messeguer X, Rozas R. 2006. DNAsp, DNA polymorphism analyses by the coalescent and other methods [J]. Bioinfor, 19: 2496-2497.

Sambrook J, Russell DW. 2002. Molecular Cloning: A Laboratory Manual. 3rd ed. [M] Huang PT, Wang JX, Zhu HC, transl. Beijing: Science Press, 463-464. [萨姆布鲁克 J, 拉塞尔 DW 著. 2002. 分子克隆实验 指南. 3 版. 黄培堂, 王嘉尔, 朱厚础等译. 北京: 科学出版社, 463-464.

Santos S, Hrbek T, Farias IP, Schneider H, Sampaio I. 2006. Population genetic structuring of the king weakfish, Macrodon ancylodon (Sciaenidae), in Atlantic coastal waters of South America: Deep genetic divergence without morphological change [J]. Mol Ecol, 15: 4361-4373.

Thompson JD, Gibson TJ, Plewwniak F, Plewniak F, Jeanmougin F, Higgins DW. 1997. The Clustal-X windows interface: Flexible strategies for multiple sequences alignment aided by quality analysis tools [J]. Nucl Acids Res, 25: 4876-4882.

Verheyen E, Salzburger W, Snoeks J, Meyer A. 2003. Origin of the superflock of cichlid fishes from lake Victoria, East Africa [J]. Science, 300: 325-329.

Vrijenhoek RC. 1994. Genetic diversity and fitness in small populations[A]. In: Loeschcke V, Tomiuk J, Jian S K eds. Conservation Genetics [M]. Basel: Birkhäuser, 37-53.

Wright S. 1978. Evolution and the Genetics of Populations [A]. Vol. 4: Variabilty Within and Among Natural Populations[M]. Chicago: University of Chicago Press.

Wang WW, Zhao JL, Li SF. 2006. Gennetic variation of the mitochondrial DNA Cyt $b$ among six popuplations of Siniperca scherzeri in China [J].
Zool Res, 27(6): 589-593. [王伟伟，赵金良，李思发. 2006. 我国斑鲊 六个群体 mtDNA Cyt $b$ 序列的遗传变异. 动物学研究, 27（6）: 589-593.]

Wang ZQ, Yang JQ, Tang WQ. 2006. A long-term misidentified new record species of gobiidae from China-Periophthalmus magnuspinnatus [J]. Acta Zootax Sinica, 31(4): 906-910. [王正琦, 杨金权, 唐文乔. 2006. 一种长期被误鉴的中国新记录鱼类一大鯺弹涂鱼. 动物分类学报, 31 (4) : 906-910.]

Xiao WH, Zhang YP, Liu HZ. 2001. Molecular systematics of Xonocyprinae (Twleostei: Cyprinidae): Taxonomy, biogeography and co-evolution of a special group restricted in East Asia[J]. Mol Phyl Evol, 18(2): 163-173.

Yeh JC, Yang R, Boyle T. 1999. POPGEME, version 1.31, Microsoft window-based freeware for population genetic analysis.

Yu ZN, Kong XY, Guo TH, Jiang YY, Zhuang ZM, Jin XS. 2005. Mitochondrial DNA sequence variation of Japanese anchovy Engraulis japonicus from the Yellow Sea and East China Sea [J]. Fish Sci, 71: 299-307.

Zhang QY, Hong WS. 2006. Review and prospect of mudskipper Boleophthalmus pectinirostris studies [J] J Xiamen Univ, 45(Sup 2) : 97-108. [张其永, 洪万树. 2006. 大弹涂鱼研究的回顾与展望. 厦门 大学学报(自然科学版), 45(增刊 2): 97-108.]

Zhu SH, Zheng WJ, Zou JX, Yang YC, Shen XQ. 2007. Mitochondrial DNA control region stucture and molecular phylogenetic relationship of carangidae[J]. Zool Res, 28(6): 606-614.[朱世华, 郑文娟, 邹记兴, 杨迎春, 沈锡全. 2007. 鯵科鱼类线粒体 DNA 控制区结构及其系统 发育关系. 动物学研究, 28 (6) : 606-614.]

\section{《动物学研究》被评选为 2008 年度中国精品科技期刊}

据中国科学技术信息研究所《2007 年度中国科技论文统计结果》, 《动物学研究》经过精品科技期刊 遴选指标体系综合评价, 被评选为 2008 年度中国精品科技期刊。

国家科技部自 2000 年以来，先后立项进行了“中国精品科技期刊战略研究”和 “中国精品科技期刊 服务与保障系统” 两个课题, 为提升中国科技期刊的整体水平, 提出了打造精品科技期刊的概念。精品科 技期刊服务与保障系统领导小组由两位院士担任项目组长, 领导小组成员来自国家科技部、新闻出版总署、 中宣部、卫生部、中国科协、国家自然科学基金委、国家教育部等科技期刊的管理部门。

按照精品科技期刊战略的目标, 根据目前我国学术期刊的发展状况以及精品科技期刊战略的总体目 标，将精品科技期刊的对象分为两类，一类是国际化精品科技期刊; 一类是中国精品科技期刊。

中国精品科技期刊的遴选指标由定量指标和定性指标两部分组成, 遴选时以定量指标为主, 定性指标 为辅。定量指标主要包括学术质量水平指标和国际竞争力水平指标。定性指标主要是指期刊的可持续发展 潜力指标。

首批精品科技期刊由 Acta Mechanica Sinica 等 23 种英文版中国国际化精品科技期刊和《中华医学 杂志》等 300 种中国精品科技期刊组成。 